\title{
Análisis del impacto del fenómeno del Monzón en la producción agrícola de la India
}

María Guillermina Diaz ${ }^{1}$

\section{Artículo científico}

Material original autorizado para su primera publicación en el Journal de Ciencias Sociales, Revista Académica de la Facultad de Ciencias Sociales de la Universidad de Palermo.

Recibido: 24-11-2015

Aceptado: 13-12-2015

\section{Resumen}

La producción agrícola de la India se ve afectada por el fenómeno del Monzón, término que significa "estación", lo cual explica su aplicación a los cambios estacionales a gran escala del régimen de los vientos y la alternancia de un período seco y otro húmedo.

El objetivo del presente trabajo consiste en analizar el fenómeno del Monzón y su relación con la anomalía del Pacífico o fenómeno del Niño (ENOS), para dar a conocer el impacto de dichos eventos climáticos sobre la actividad agrícola de la India.

Se utiliza el método geográfico, que comprende tres etapas: la primera es la observación indirecta, realizada a través del análisis de datos cuantitativos (como datos pluviométricos del fenómeno del Monzón y del ENOS en diferentes períodos), como así también datos cualitativos, donde se trabaja la relación entre rendimiento de producción agrícola y el abastecimiento de alimentos de la población. La segunda etapa, la explicación, donde se presenta un análisis de los datos observados y trabajados, en relación con la alimentación de la población de la India. Y por último, la síntesis, mediante cartografía representativa.

Finalmente, lo anteriormente planteado no discrimina los impactos negativos que se generan en la población sobre la base del fenómeno del Monzón, cuando ocurre alguna anomalía como el ENOS.

Palabras clave: monzón, fenómeno del Niño, agricultura, India.

\section{Abstract}

Agricultural production in India is affected by the phenomenon of the monsoon, which means season, which explains its application to large-scale seasonal wind regime changes and the alternation of a dry and a wet period.

The aim of this essay is to analyze the impact of monsoon on agricultural activity in India and its relationship to the anomaly of the Pacific or El Niño (ENSO), which affects much of Latin America.

1 Departamento de Geografía. Profesora en Geografía. Cargo: Ayudante de Segunda Ad-Honorem. Correo electrónico: guillerminadiaz07@gmail.com 
It is used the geographic method, that comprises three stages: the first is the indirect observation which is made through the analysis of quantitative data (such as rainfall data of the phenomenon ENSO and Monsoon in different periods), as well as qualitative data, where the relationship will work between performance of agricultural production and food supply of the population. The second stage, the explanation, where an analysis of observed and worked data in conjunction with the feeding of the population of India data is presented. Finally, the synthesis, by representative cartography.

To conclude, all the above specified does not discriminate negative impacts generated in the population based on the phenomenon of the monsoon, when an abnormality occurs as ENSO.

Key words: monsoon, El Niño, agriculture, India.

\section{Introducción}

La actividad agrícola de la India es la principal dentro de la economía del país, es por ello que realizar un análisis del impacto del monzón sobre ella puede ser importante para poder lograr una correcta planificación de dicha actividad, teniendo en cuenta los efectos negativos que se desencadenan sobre la base del fenómeno del monzón en la población.

Sin embargo, las variaciones climáticas que dicho fenómeno provoca no son la única causa que afecta la población, sino también las diferencias a nivel regional y las cuestiones políticas tienden a generar diferencias de tipo socio-económico entre las regiones.

El objetivo del presente trabajo consiste en analizar el fenómeno del Monzón y su relación con la anomalía del Pacífico o fenómeno del Niño (ENOS), para dar a conocer el impacto de dichos eventos climáticos sobre la actividad agrícola de la India.

Se utiliza el método geográfico propuesto por Ostuni (1976), cuyos pasos son los siguientes: 1) Captación del hecho: primer contacto con la realidad, se apoya en la observación directa (encuesta, trabajo de campo, entrevistas, entre otras) y observación indirecta (documentos cartográficos, bibliografía general y específica, fotografías aéreas, imágenes satelitales, entre otras); 2) Análisis geográfico: se busca lo relevante del cuadro natural, la población y sus recursos, los recursos y su utilización, la circulación y los polos de actividad, y luego se procede a: la interpretación de cartas, la elaboración de cartogramas, gráficos y cuadros analíticos y la recopilación estadística; 3) Síntesis geográfica: se logra la correlación de los elementos del análisis al establecer la estructura geográfica del espacio investigado, que se expresa en el croquis sintético y la descripción explicativa; y 4) Diagnóstico prospectivo: se tiende a una racional ocupación del espacio al presentar situaciones y los juegos de fuerza, y al integrar equipos interdisciplinarios de planificación a nivel de decisiones. Finaliza en la evaluación crítica del espacio. 
De los pasos del método planteado con anterioridad se seleccionaron los siguientes para desarrollarlos a lo largo del trabajo: Captación del hecho: primer contacto con la realidad a través de la observación indirecta (documentos cartográficos, bibliografía general y específica, fotografías aéreas, imágenes satelitales, entre otras); 2) Análisis geográfico: se busca lo relevante, el cuadro natural, la población y sus recursos, los recursos y su utilización, la circulación y los polos de actividad. Luego se procede a: la interpretación de cartas, la elaboración de cartogramas, gráficos y cuadros analíticos y la recopilación estadística; 3) Síntesis geográfica: se logra la correlación de los elementos del análisis al establecer la estructura geográfica del espacio investigado, que se expresa en el croquis sintético y la descripción explicativa.

En una primera parte se muestra la localización geográfica del área en estudio, se explica el fenómeno del Monzón en las estaciones de verano y de invierno respectivamente. En una segunda parte, con soporte de datos cuantitativos se realiza un análisis de dicho fenómeno y se presenta la cartografía para el mismo. Una tercera parte comprende la explicación del desarrollo de la anomalía del Niño y su relación con el Monzón, y a su vez este con la producción agrícola por regiones. Por último, mediante cartografía se representan las regiones de los principales cultivos que se ven afectadas por el Monzón de verano.

Localización del área de estudio

\section{Localización Geográfica de la India}

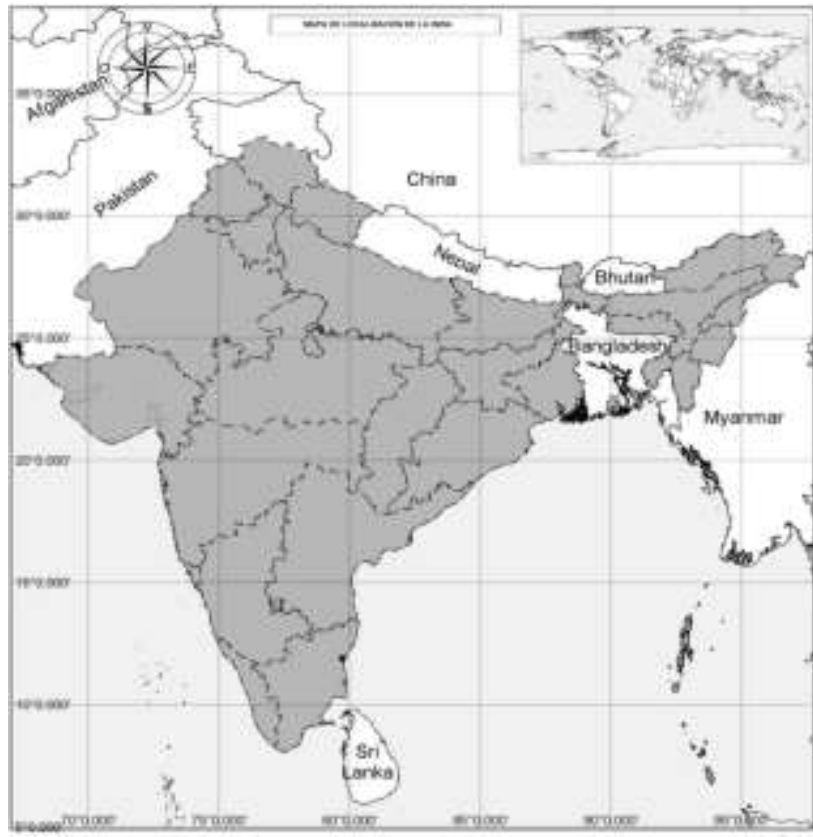

Elaboración propia sobre material de ArcGIS.

Dibujo: Manuel Maffini (2015) 
La India tiene una posición absoluta comprendida entre los $66^{\circ} \mathrm{E}$ y $98^{\circ} \mathrm{E}$, y $\operatorname{los} 5^{\circ} \mathrm{N}$ y $35^{\circ} \mathrm{N}$. En cuanto a su posición relativa, se puede decir que es un país peninsular que se sitúa en el Hemisferio Norte, al sur de Asia comprendiendo un área superficial de 3.000 .000 de km2 y 7000 km2 de línea de costa aproximadamnte, entre Bangladesh y Paquistán sobre la Bahía de Bengala, el Mar Arábigo y el Océano Índico, con los cuales limita en su parte este, oeste y sur respectivamente. La India asimismo limita al norte con la cordillera del Himalaya.

Topográficamente está conformada por grandes planicies localizadas al Norte, separadas del sector sur por la cadena montañosa Vindhya, situada esta en el centro del país. El relieve de la zona central y sur esta conformado principalmente por mesetas de poca altura, Ilanuras costeras. Hacia el suroeste se hallan algunas cadenas montañosas que son importantes para la distribución de las precipitaciones.

La topografía actúa como un factor determinante del clima, así por ejemplo la cordillera del Himalaya funciona como una barrera a los vientos procedentes de Asia Central, siendo ello un elemento fundamental en las regiones del Norte donde el clima es seco con inviernos fríos y veranos calurosos. Sin embargo, en las regiones costeras las temperaturas son más elevadas con poca variación durante el año y el régimen pluviométrico es más abundante. Esta diversidad climática está muy influenciada por el fenómeno del Monzón.

El término Monzón deriva del vocablo árabe mausim, que significa estación. Dicho término se utiliza para indicar cualquier ciclo climático anual con la inversión estacional de los vientos dominantes en las regiones tropicales y subtropicales, dando origen a cambios estacionales tanto de la circulacion atmosférica como de la precipitación asociada.

El clima monzónico se caracteriza principalmente por la oposición de un período seco y fresco, conocido como el Monzón de invierno, a un período húmedo y cálido correspondiendo este al denominado Monzón de verano. Se produce un sistema acoplado océano-atmósfera y su fuerza esta determinada por los procesos de interacción airemar, siendo el resultado del contraste entre el calentamiento de la superficie terrestre y del océano, dando origen a importantes variaciones en la presión atmosférica, temperatura y vientos. Este contraste se produce porque las superficies marinas almacenan y transportan energía de manera más eficiente que la superficie terrestre, ya que una masa de agua extensa y profunda se calienta y se enfría de forma mucho más lenta que un cuerpo sólido, como lo es la superficie terrestre. Explica al respecto Hoyos Ortiz:

En las horas de la mañana, antes de la salida del sol, la temperatura superficial del océano y del continente es muy similar y las isobaras tienden a estar paralelas entre sí y con la superficie. En el trascurso del día la tierra se calienta más rápidamente que el océano debido a los factores anteriormente mencionados, calentando la atmósfera que reposa sobre ésta. Como respuesta a tal calentamiento, la atmósfera sobre la superficie terrestre se expande creando un gradiente de presión horizontal en el nivel superior, generando flujo de aire hacia el océano. Dado que la presión superficial en un lugar está determinada por el peso del aire sobre éste, 
la remoción de aire en cualquier nivel reduce la presión superficial sobre la tierra, estableciéndose un nuevo gradiente de presión que genera flujo superficial de aire hacia la tierra. Posteriormente, movimientos verticales son inducidos por los horizontales para cumplir la ecuación de continuidad (Hoyos Ortiz, 2003, p. 5)

De esta manera, para conservar el balance de energía, se da una transferencia de calor desde las zonas más calientes hacia las más frías, formando lo que se llama la circulación meridional del Monzón.

Algunas de las características que hacen del Monzón de la India un fenómeno particular son la gran cantidad de superficie terrestre que fortalece el calentamiento diferencial, la presencia de la meseta del Tibet que perturba la respuesta atmosférica al calentamiento solar, la alta cantidad de humedad disponible debido a la presencia del Mar Arábigo y del Océano Índico, y la existencia del chorro Somalí que transporta humedad.

\section{Desarrollo del Monzón de verano y de invierno}

Durante el verano boreal, a principio del mes de junio, se produce la brusca invasión del monzón de verano, que se ve acompañado por una leve disminución de la temperatura. El alisio austral que cambia la dirección como consecuencia de la actuación de la fuerza de coriolis, cuando atraviesa el ecuador atraído por los centros de bajas presiones situados al Norte de la India, tiene un espesor considerable y es el que nutre el flujo del Monzón. A pesar de que dicho flujo es fuerte y regular, cuando el relieve se interpone la masa de aire se ve forzada a elevarse produciendo una mayor cantidad de precipitaciones; lluvias orográficas de la costa oeste de la Península del Dekán, lluvias ligadas al estrechamiento del corredor de Assam.

Además del relieve, el flujo del Monzón también se ve afectado por otras perturbaciones que provienen del Mar Arábigo, otras se originan sobre el Golfo de Bengala, las ligadas a la circulación del este que permanecen en altura por encima del flujo del Monzón y las invasiones frías de altitud, capaces de acentuar la convección.

El Monzón, que ingresa por el sector oeste a la India, se incurva por encima de la llanura Indo-Gangética continuando el valle depresionario o talweg del Monzón, siempre ubicado al Sur del Himalaya y cuya posición en latitud parece determinar precipitaciones. Dicho flujo cuando alcanza la zona de Punjab llega empobrecido, y prácticamente no afecta al oeste del Himalaya.

La corriente del Monzón es muy sensible a las influencias del relieve, la meseta del Dekán está al abrigo de los Gaths Occidentales, siendo irregular de un año a otro sobre la mayor parte de la India, como cuando se produce el retardo del Monzón, dando lugar a sequías prolongadas. 
Barry y Chorley afirman: "En la ladera a sotavento de los Ghats, las cantidades son mucho menores y existen zonas semiáridas que reciben menos de 64 cm por año" (1999, p. 279).

La configuración de la tierra y el mar juegan un rol fundamental, principalmente en el Golfo de Bengala. En otoño, cuando el Monzón ya se retiró, las depresiones nacidas en el golfo son las que aportan aire húmedo hacia el Dekán Oriental, donde las lluvias tienen su máximo estacionario. Asimismo el Himalaya constituye para el Monzón de la India una barrera inaccesible, los valles interiores de abrigo son subdesérticos siendo la agricultura posible sólo a través del riego, con el agua que proviene del derretimiento de nieve y glaciares. El oeste del Himalaya está poco influenciado por el Monzón de verano.

Cabe destacar que el Monzón de verano aporta el $80 \%$ de las lluvias anuales a la India.

La situación durante el invierno es distinta de lo que sucede en el verano. En invierno el continente asiático está dominado por un centro de altas presiones (anticiclón siberiano), el cual es de origen térmico pelicular; las masas de aire provenientes de dicho anticiclón al desplazarse se encuentran con el cordón montañoso del Himalaya, que debido a su altura, actúa como barrera al paso de las masas de aire del anticiclón, no permitiendo el ingreso del aire siberiano sobre la India.

Las depresiones que rodean al Himalaya están relacionadas al Jet- Stream que pasa entonces al sur de la cadena montañosa; las mismas aportan al noroeste de la India aire fresco y húmedo con algunas precipitaciones favorables al cultivo del trigo. El sector sur del país es dominio del clima ecuatorial, con la influencia de la circulación del este.

El Monzón invernal trae un tiempo seco durante un largo período, produciéndose una ausencia de precipitaciones y la estación seca y fresca nombrada anteriormente, que se va recalentando paulatinamente hacia la primavera.

Las siguientes imágenes grafican lo explicado anteriormente sobre las estaciones del verano y del invierno boreal, a través de las cuales puede verse la dirección y la incidencia de los vientos, y el consecuente desarrollo de las precipitaciones sobre el área bajo estudio. 
(a) Verano boreal

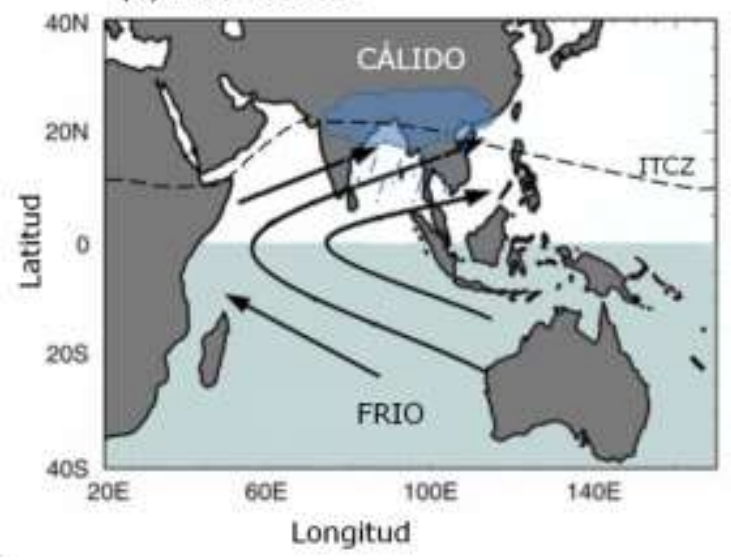

(b) Invierno boreal

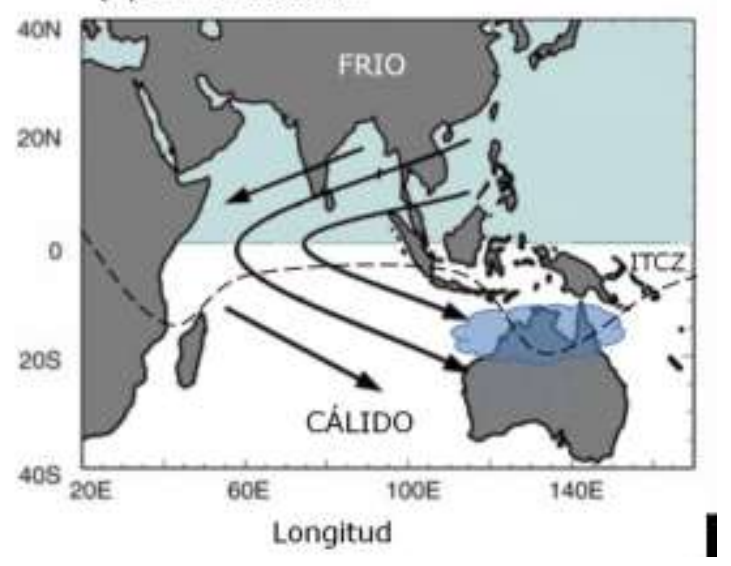

Fuente: Ordóñez Pérez (2013).

A través de datos pluviométricos registrados por el Departamento Meteorológico de la India se puede decir que la región que presenta el mayor promedio de precipitaciones caídas durante los períodos 1901-1930, 1931- 1970 y 1971-2013, en momentos del Monzón de verano, es la región noreste; y la región que presenta los valores menores de precipitación es la del noroeste, mientras que las regiones sur y centro tienen los promedios intermedios. Ello se debe a características topográficas mencionadas con anterioridad, y las que tienen que ver con la dirección del ingreso de las masas de aire hacia el continente cuando se produce el Monzón.

A su vez se puede decir que con el paso del tiempo se produjeron algunas modificaciones en la variación estacional, en cuanto a la cantidad de precipitación, teniendo una tendencia a disminuir, siendo el período $1971-2013$ el que más lo demuestra. Una de las causas que puede explicar dicha tendencia es que dentro de los tres períodos se sucedieron los años más notables del Niño, 1925 dentro del primer período, 1940-41 y 1965 en el segundo período, y 1972-73, 1982-83, 1997-98 durante el tercer período, haciendo que disminuyan las lluvias en la parte occidental del Océano Pacífico. 
La siguiente imagen da cuenta de lo explicado anteriormente:

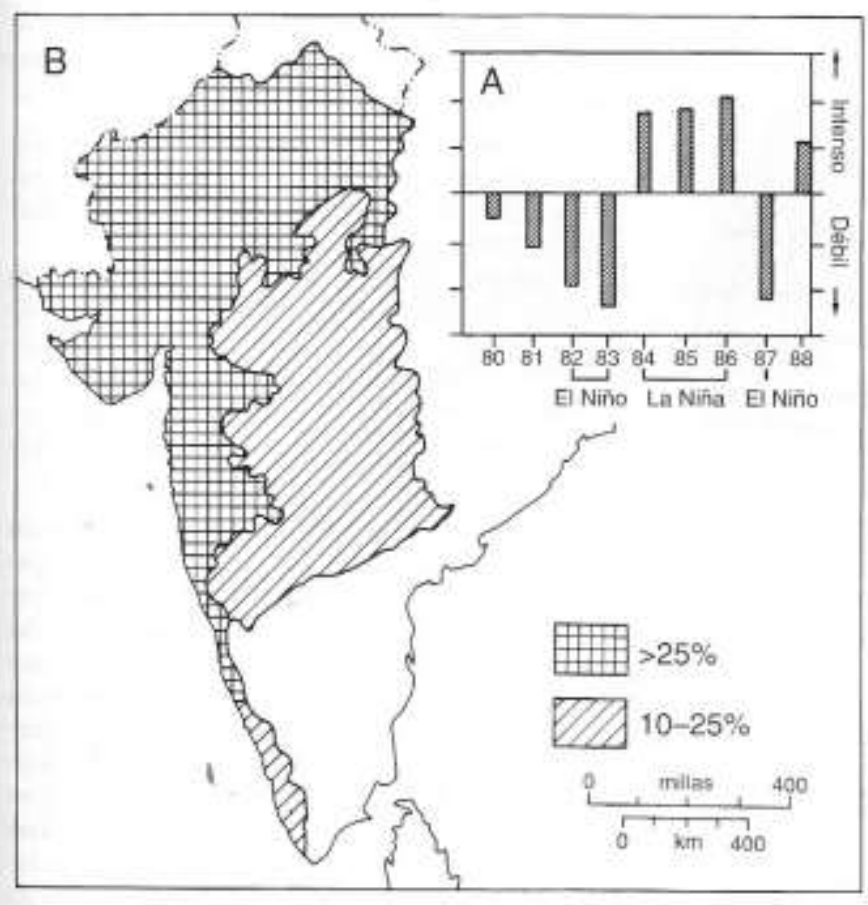

Conexión

propuesta entre el monzón de verano en la India y El Niño. A: La intensidad coservada en ei monzón de verano asiático (1980-88) mengua durante los Ires anos (1982, 1983 y 1987). en los que se manifesta $\mathrm{E}$ Niño con intensidad. B: Zonas de la India donde las déficits de precipitaciones dei monzón do verano (en un porcentaje inferior a la media de 1901-1970) presentaron una frecuencia notabiemente mayor durante los tres años de El Niño.

Fuente: Barry y Chorley (1999).

A continuación se representa lo expuesto mediante cartografía representativa de los períodos mencionados, donde puede verse la cantidad de precipitación registrada por regiones.

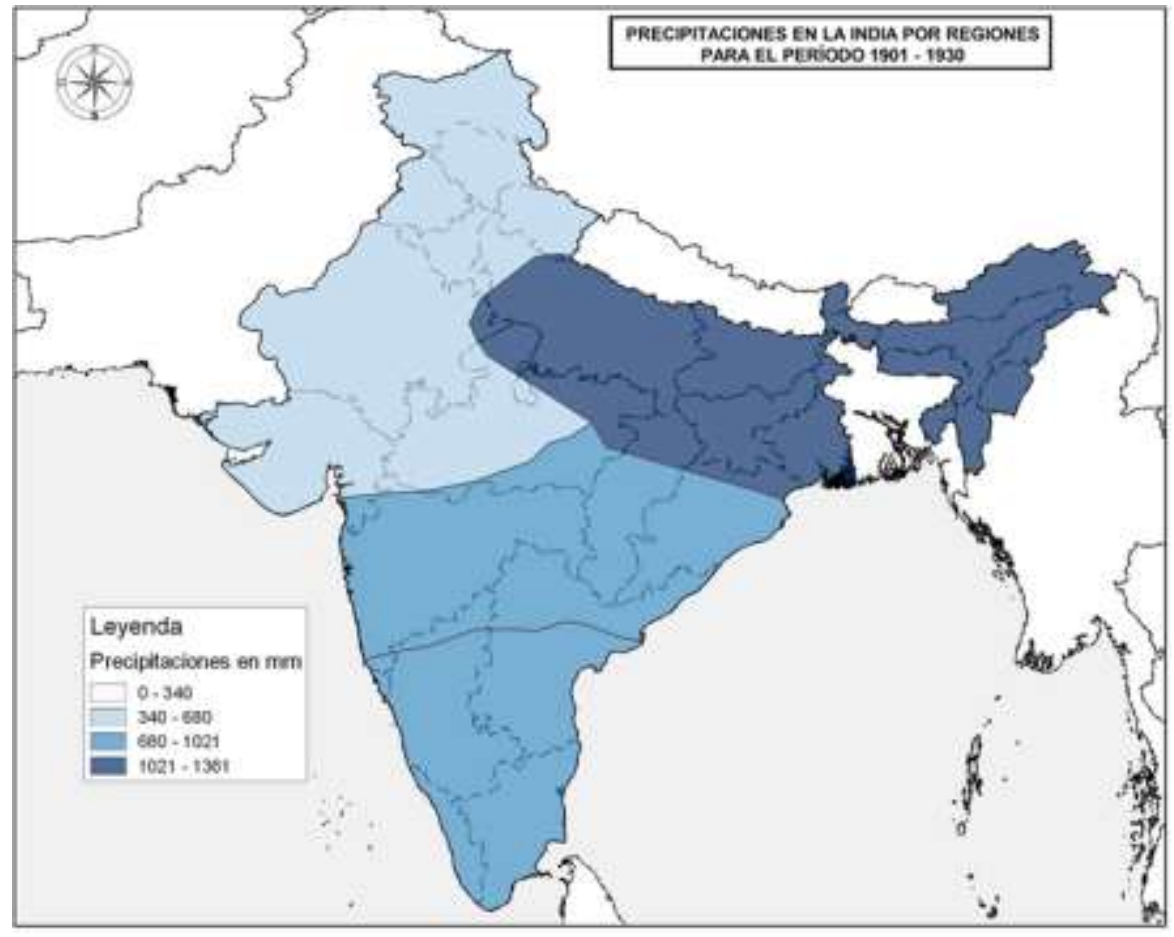

Elaboración propia sobre datos del_India Meteorological Department Dibujo: Manuel Maffini (2015) 


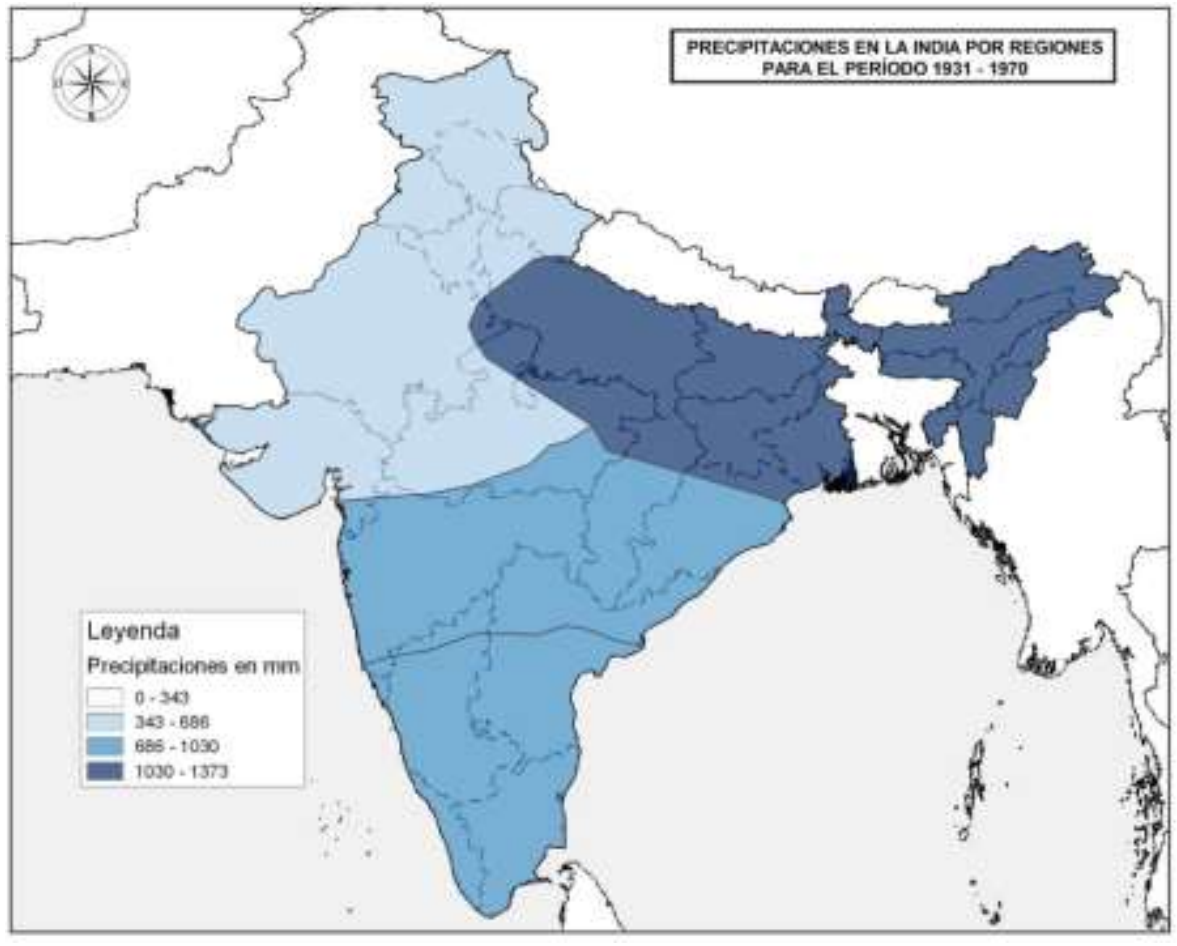

Elaboración propia sobre datos del India Meteorological Departmen Dibujo: Manuel Maffini (2015)

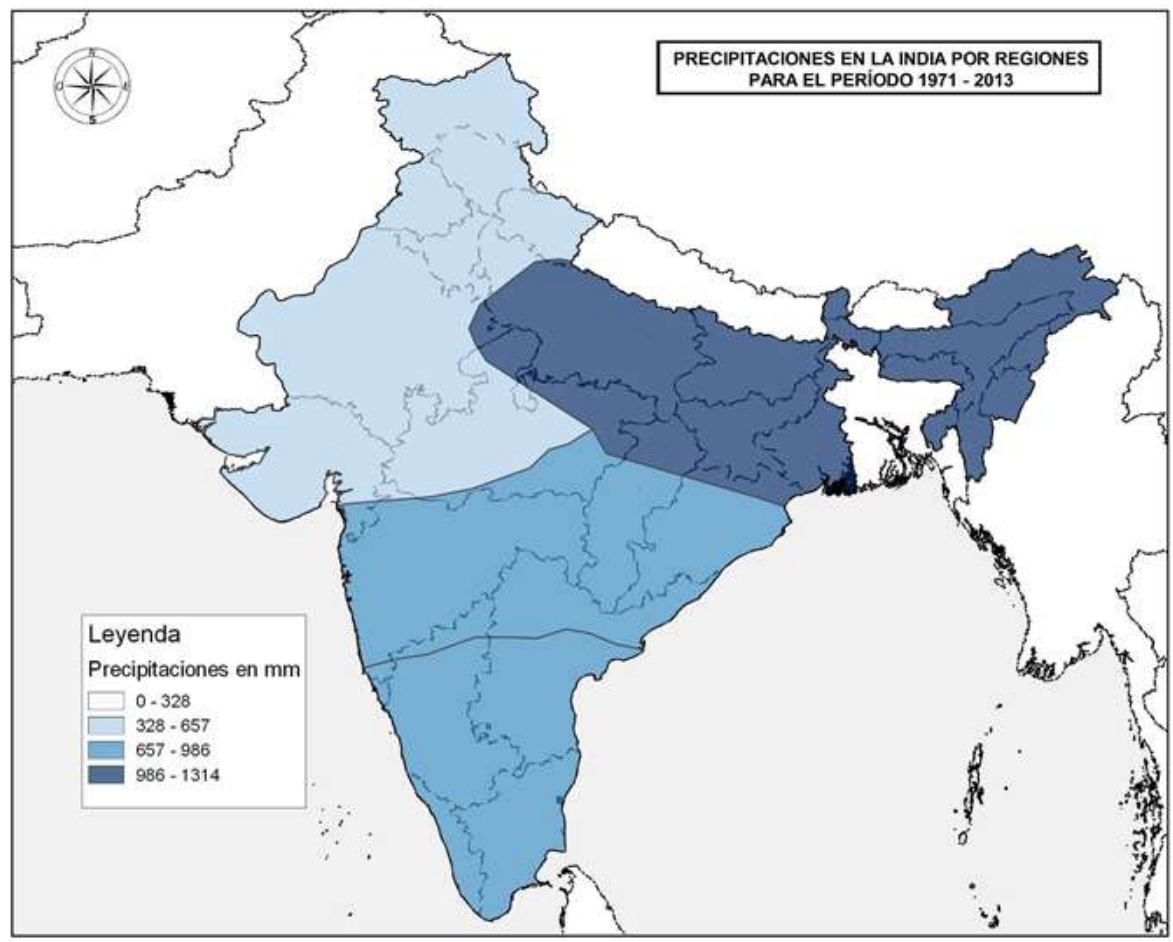

Elaboración propia sobre datos del_India Meteorological Department Dibujo: Manuel Maffini (2015) 
Además de la variación estacional expuesta anteriormente, en los índices monzónicos existe variabilidad en otras escalas temporales como lo es la escala interanual.

Siguiendo a Hoyos Ortiz (2003), la variabilidad interanual del Monzón ha sido explicada desde diferentes puntos de vista. Se ha propuesto que esta se debe al cambio lento de las condiciones de borde, determinadas principalmente por la temperatura superficial del mar, la humedad del suelo, y la cobertura de nieve sobre Eurasia; otros explican que tales variaciones se dan en la dinámica interna del fenómeno, o en su propia naturaleza.

\section{Anomalía del Pacífico: Fenómeno ENOS}

Una de las principales variaciones en las condiciones de borde se debe a los cambios que ocurren en el Océano Pacífico, y estos a su vez al fenómeno de El Niño (ENOS).

Sánchez (2002) sostiene que se conoce con el nombre de ENOS a un fenómeno de la naturaleza de efectos globales generado en el Océano Pacífico, que ocurre a intervalos de cuatro a cinco años, causando catástrofes naturales, con fuertes impactos en los ecosistemas de la región. Este fenómeno consiste en el aumento generalizado de la temperatura de la superficie del mar en gran parte del sector oriental y central del Pacífico Ecuatorial, unido a un incremento de la temperatura del aire en zonas costeras, vientos débiles, disminución de la presión atmosférica en el Pacífico Sur-Oriental, disminución del afloramiento marino, incremento del nivel del mar frente a las costas de Perú, Ecuador y Chile, y fuertes precipitaciones; estas características han de persistir por lo menos durante cuatro meses continuos.

Por razones que los científicos aún no comprenden, cada determinado número de años, los vientos alisios se debilitan o incluso desaparecen. El patrón usual de la presión del aire se invierte en un fenómeno llamado Oscilación del Sur, lo que provoca que las lecturas de los barómetros en Australia sean más altas que las del Pacífico central. El patrón resultante (conocido como ENOS, que significa EI Niño Oscilación del Sur) abarca solo una quinta parte del planeta, pero transforma el clima alrededor del globo. (Sánchez, 2002) (Arntz y Fahrbach, 1996)

La corriente del Perú o Humboldt se caracteriza por la emergencia del agua fría de las profundiades, cargada de iones nutrientes, que sirven de alimento al plancton que allí se desarrolla. Con la aparición del Niño, cesa el ascenso del agua fría siendo reeemplazada por aguas cálidas procedentes del oeste, de modo que tanto el plancton como los depredadores de la anchoveta desaparecen, apareciendo peces de tipo tropical que desplazan a la anchoveta, siendo esta parte de la actividad económica de los pescadores costeros; asimismo se producen aguaceros que pueden ser abundantes sobre la costa. 
Sánchez (2002) afirma:

Sin los vientos alisios, la capa superior del Pacífico oriental no se mueve hacia el oeste; permanece ahí, se calienta cada vez más y se expande conforme aumenta la temperatura. Durante El Niño 1997-98, los niveles del mar, cerca de América del Sur aumentaron $25 \mathrm{~cm}$. arriba del nivel normal y las temperaturas superficiales alcanzaron casi los $30^{\circ} \mathrm{C}$. Cuando esto sucede, el agua en la atmósfera superior se condensa y se precipita como lluvia torrencial sobre la costa oeste del continente americano. Esto a su vez, reduce la salinidad de los mares costeros, donde la corriente ascendente de las aguas profundas ha disminuido o se ha detenido. Debido a que El Niño lleva hacia el continente americano las lluvias que normalmente caerían en el Pacífico Occidental, lugares como Australia, Indonesia e India pueden experimentar serias sequías. (2002, p.120)

Se puede decir que El Niño (ENOS) es una anomalía climática ya que dentro de un esquema zonal se refiere a una ruptura local con respecto a las condiciones medias que caracterizan a la zona en que se sitúa el fenómeno antes mencionado. De este modo, se ha relacionado la ocurrencia de eventos de El Niño con la deficiencia del Monzón. El desplazamiento de la convección sobre el oeste del Pacífico hacia el este, particularidad asociada con El Niño, produce un movimiento descendiente en la tropósfera sobre la India disminuyendo la cantidad de lluvia.

Cuando las presiones se incrementan en Australasia y simultáneamente disminuyen en el Sudeste del Océano Pacífico, se producen en la costa occidental del Pacífico procesos como el aumento de la presión atmosférica, la disminución de la temperatura del agua y por lo tanto del aire que está por encima, disminución del nivel del mar y sequías generalizadas. Es así que el Monzón de verano de la India queda debilitado o no se desarrolla, originándose eventuales sequías. En cambio, cuando la oscilación barométrica en el Pacífico es inversa, el Monzón se refuerza produciendo abundantes precipitaciones.

La interacción entre el ENOS y el Monzón se produce por el efecto que ejerce la temperatura superficial del Océano Pacífico en la localización e intensidad de la ascendencia- subsidencia de la circulación atmósferica ecuatorial (circulación de Walker) que a su vez influye sobre la circulación Norte-Sur regional asociada al Monzón (circulación de Hadley).

En los meses correspondientes a la estación de invierno, el efecto que provoca El Niño sobre la India es el aumento de la temperatura, que pasa a ser más caliente de lo normal. En cambio durante el verano el efecto es el debilitamiento de la circulación del Monzón y, por ende, la disminución de las precipitaciones.

Los efectos producidos por el ENOS sobre los componentes principales del clima, cambiaron los patrones climáticos principalmente en el Pacífico ecuatorial y en todo el mundo. 


\section{Impacto del Monzón en la producción agrícola de la India}

Las variaciones que se pueden dar en el fenómeno del Monzón producidas por el ENOS influyen de manera directa sobre los cultivos producidos en las distintas regiones de la India. Es por ello que dicho fenómeno determina mediante sus dos estaciones la vida agrícola de la India. Así se pueden clasificar los cultivos de verano (jarif) y los cultivos de invierno (rabi), correspondientes a las estaciones homónimas.

La región del Nordeste indio está compuesta por los Estados de Calcuta y Bengala. A su vez se pueden distinguir las llanuras que comprenden el Assam propiamente dicho, siendo una franja de tierras bajas y pantanosas que conforma el mayor conjunto deltaico de la India, constituido por el Brahmaputra y los deltas de Bengala del Orissa. Alrededor de estas llanuras se extiende una faja montañosa, al norte el Himalaya central, al este las cadenas de Arakan, y al centro la meseta de Shillong.

Todo el conjunto regional del Nordeste tiene un régimen pluviométrico elevado, caracterizado por una estación húmeda larga. En Bengala, las pequeñas lluvias de abril y mayo permiten el adelanto de una estación agrícola - Es así que, contando esta estación, dicho Estado tiene tres cosechas anuales y por ende la estación agrícola más larga.

Al este de Bengala se encuentra una llanura que queda sumergida durante varios meses cuando se producen precipitaciones abundantes, de este modo los pueblos quedan aislados sobre colinas que se transforman en islas durante el Monzón. Bajo estas condiciones naturales el arroz flotante es el cultivo que mejor se adapta.

En la faja montañosa nombrada con antelación se práctica una agricultura con doble cosecha anual que se realiza en los valles interiores; en verano, los arrozales predominan en los valles y el maíz sobre las terrazas; y en invierno el trigo y la cebada, sin olvidar las plantaciones de té en los márgenes montañosos.

La llanura Gangética y el Himalaya central son las dos regiones regadas por el río Ganges. Al este de Bihar el clima es de tipo bengalí (cinco a seis meses de sequía), al oeste de Uttar Pradesh es notablemente más seco (siete a ocho meses de sequía), pero con un invierno más fresco en donde la temperatura media es de $-15^{\circ} \mathrm{C}$. $\mathrm{El}$ clima monzónico impone a la agricultura de estas regiones condiciones rigurosas, suplidas a veces por el regadío; a pesar de que este sistema no sea indispensable para la producción agrícola, el aporte de agua del Himalaya cumple un rol fundamental. Explica a su vez ciertas diferencias entre las regiones ubicadas al norte y al sur del Ganges. En Bihar, los campos se encuentran tradicionalmente expuestos a inundaciones, a diferencia de lo que sucede con el aprovechamiento de las aguas provenientes del Himalaya, que prometen a los productores un porvenir agrícola mejor. En general la irrigación beneficia a una cuarta parte de las tierras cultivadas (Dupuis, 1975). 
En Uttar Pradesh la agricultura sigue el calendario agrícola clásico, alternando cultivos de verano con cultivos de invierno. Asimismo en Bihar la agricultura tiene un calendario parecido al de Bengala con tres cosechas, entre los meses de mayo y julio, y entre agosto y septiembre. Regionalmente, el cultivo de subsistencia es el trigo y se cultiva por lo general en los interfluvios, con lo cual depende primordialmente de las lluvias de invierno.

El arroz caracteriza a los distritos que bordean al Himalaya, sobre todo los de Bihar, pero su cultivo es mínimo en las zonas poco irrigadas. Las densidades demográficas reflejan el carácter más o menos intensivos de los cultivos.

La región del noroeste indio está conformada por la cuenca del río Indo. Tiene una tendencia árida, por la cual la influencia del Monzón de verano disminuye, pero la tendencia mediterránea se va acrecentando cada vez más hacia el oeste de dicha región. En la cuenca del Indo se forman oasis, el oasis de Landakh, el oasis del Indo Medio, y los oasis inferiores, en los cuales se cultiva trigo y cebada, trigo, maíz, legumbres y albariqoques, arroz, maíz y mijo respectivamente. Tiene un piso agrícola lluvioso. La llanura alta de Kashmir asocia al arroz y al maíz, ambos cultivos de verano, con los cultivos hortícolas. La alternancia de dos cosechas es la base del noroeste al igual que la región del norte de la India. El trigo sembrado en octubre y diciembre y cosechado en marzo y abril por lo general no recibe las Iluvias necesarias; es por ello que dos tercios de la superficie están irrigados. El sorgo y el mijo están destinados a las regiones secas no irrigadas, y el maíz a las áreas de piedemonte donde las lluvias son más intensas.

A tráves del tiempo el desarrollo de la irrigación permitió la extensión del arroz en las zonas bajas de Punjab, y el algodón se ha convertido en el principal cultivo del noroeste; dicho sistema ha hecho que la producción agrícola dependa completamente de él, así se ha podido desarrollar el mismo gracias a la abundancia de las aguas del Indo y sus afluentes, construyéndose presas a lo largo del tiempo para receptar las aguas vertidas por el Monzón, para luego ser distribuídas en los cultivos de invierno. Pero es de destacar que la irrigación también plantea problemas, como son las infiltraciones de agua en los canales que producen el aumento del nivel de la capa freática, y además el agua cuando se estanca forma una costra salina a raíz de su evaporación.

Continuando con la división regional de Dupuis (1975), la región central de la India alcanza los Estados de Madhya Pradesh, el este y el centro de Orissa, el sur de Bihar y algunos distritos de Uttar Pradesh. En esta región, además de algunas áreas de cultivo de arroz intensivo, los productores realizan una agricultura de secano, ello se debe a las características climáticas y edafológicas, en donde los régimenes pluviométricos son bajos y los suelos poseen una proporción alta de hierro y capas de humus sensibles a la erosión. Al no retener el suelo grandes volúmenes de agua, los cultivos de invierno no pueden producirse debido a que durante la estación seca las lluvias son escasas. Asimismo, los agricultores realizan una producción con una gran cantidad de barbecho prolongado (por cada año de cultivo, tres o cuatro años de barbecho). 
Se puede decir que la región central no tiene una producción agrícola uniforme, sino que la misma es el producto de la transición entre las regiones que las rodean, teniendo como característica propia y general, la pobreza.

Maharashtra y Gujrat son dos Estados situados hacia el oeste del país, conforman una unidad cultural y económica pero presentan diferencias en cuanto a cuestiones de índole físicas. El primero está más expuesto al flujo del Monzón, de tal modo que registra una mayor pluviosidad que Gujrat. En este último se practica una agricultura similar a la del noroeste con irrigación mediante embalses y pozos. En las llanuras el arroz es el cultivo esencial, pero también se realizan los cultivos de trigo en rabi y mijo en jarif.

En Maharashtra el cultivo por excelencia es el mijo, le sigue el sorgo, el algodón, el tabaco y la caña de azúcar.

Las regiones agrícolas de ambos estados brindan un conjunto variado donde tienen lugar complejas transiciones entre las formas agrícolas del noroeste y las del Dekán central y meridional. Esta base rural es por lo general pobre, sin embargo, ciertas disposiciones de la naturaleza o de la sociedad han facilitado localmente áreas de cultivos que son comercializados y muy productivos (Dupuis, 1975).

En los macizos montañosos de la región meridional, los productores se dedican a las plantaciones. En la llanura occidental de Kerala la producción agrícola está compuesta por los cultivos de especias, arecas, cocoteros y arroz.

En el sudeste de la India se practica una agricultura de secano en las zonas interfluviales, y una agricultura intensiva en las zonas levemente hundidas; teniendo un rol fundamental los sistemas de riego, al actuar las grandes presas como grandes depósitos que acumulan el agua de las crecidas. 
DIVISIÓN POLÍTICA DE LA INDIA

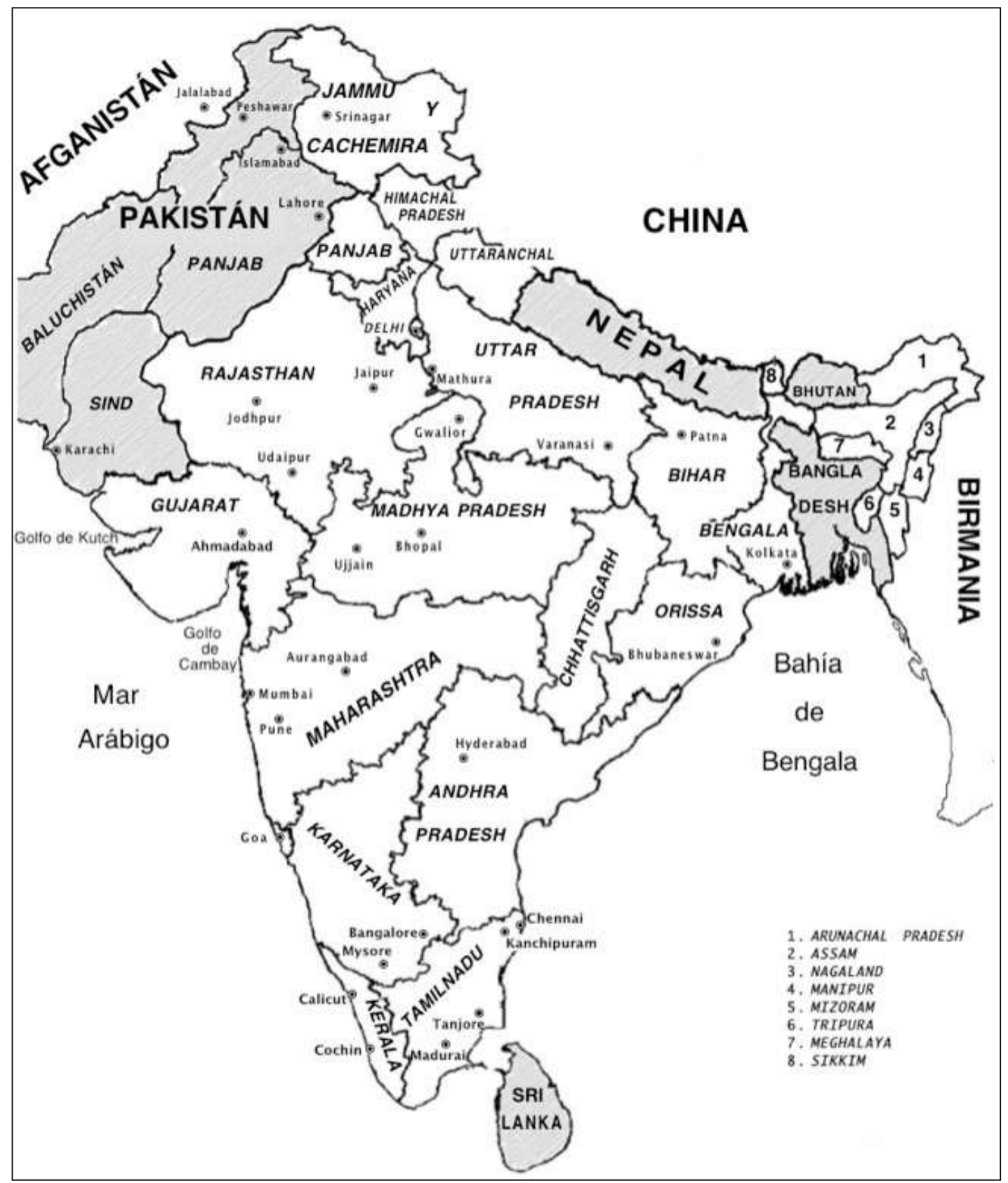

Fuente: http://www.elportaldelaindia.com 


\section{SINTESIS CARTOGRÁFICA}

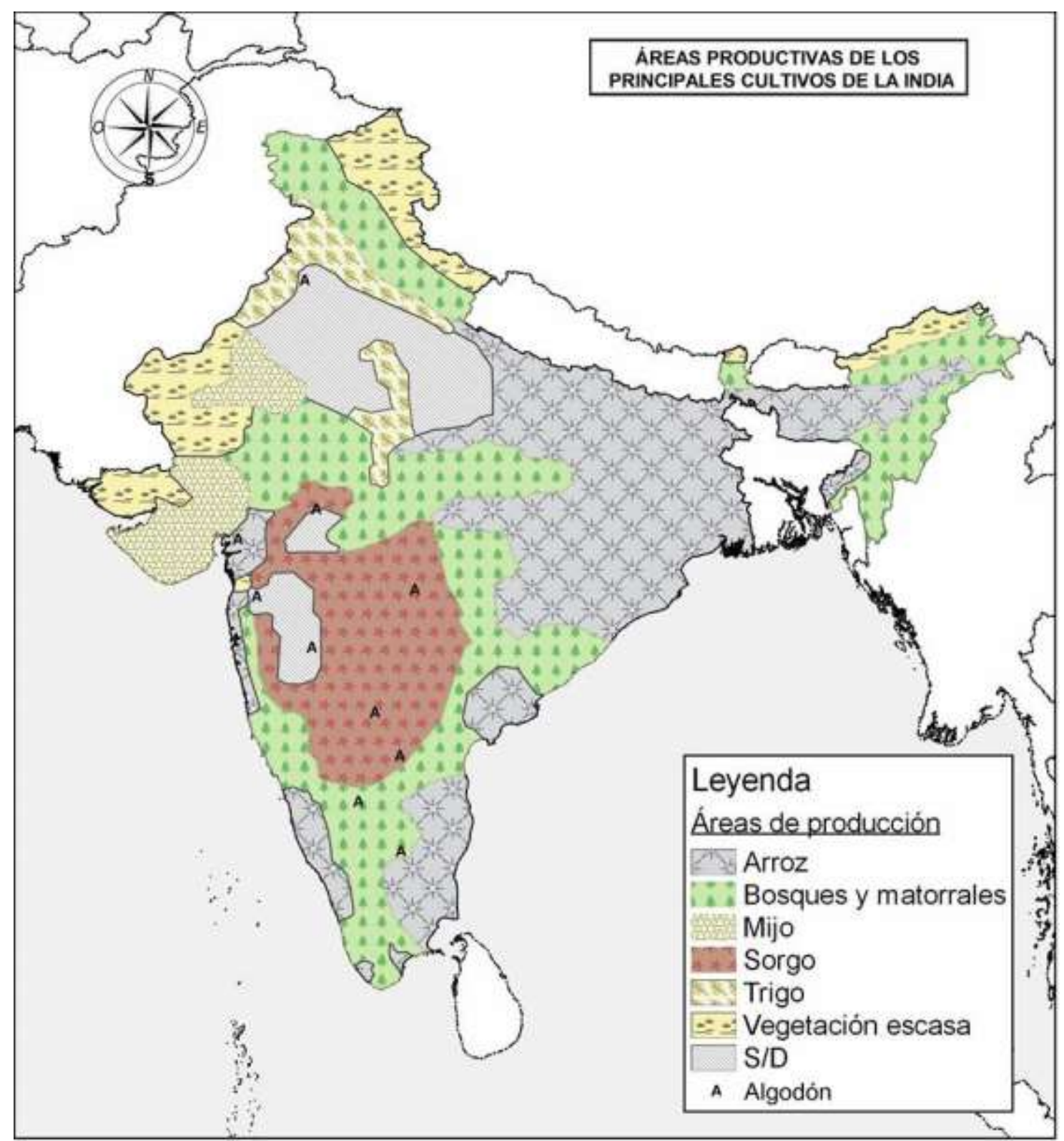

Elaboración propia sobre_Dupuis (1975)

Dibujo: Manuel Maffini (2015) 


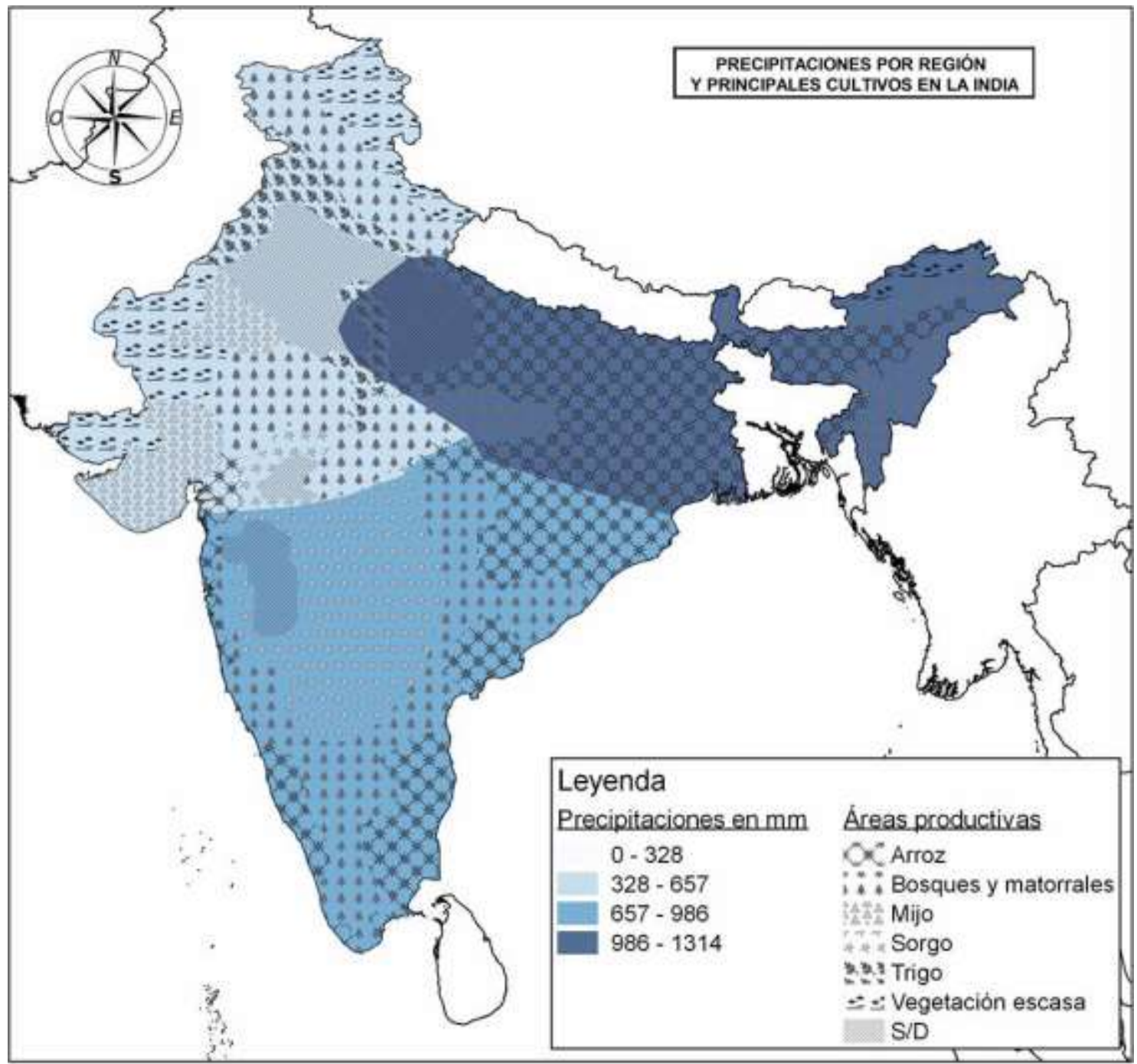

Elaboración propia sobre datos del India Meteorological Department Dibujo: Manuel Maffini (2015)

Se puede decir que las regiones más afectadas por las variaciones climáticas y el hambre son las secas, y las regiones húmedas están mejor protegidas contra esas variaciones; sin embargo, como son también las más pobladas, su producción alimenticia es gravemente deficitaria.

La lucha en el terreno alimenticio está relacionada con los diferentes niveles de encauzamiento y utilización del agua, y con las mejoras locales.

Alrededor de un $70 \%$ de las familias rurales y un $8 \%$ de las familias urbanas siguen dependiendo principalmente de la agricultura como fuente de trabajo. Habida cuenta de que las tres cuartas partes de la población viven en zonas rurales, la mayoría de las familias indias dependen primordialmente de este sector. 


\section{Consideraciones finales}

Las variaciones climáticas provocadas por la influencia del ENOS sobre el Monzón provocan en la actividad agrícola consecuencias importantes, ya sean positivas o negativas, entre estas últimas, sequías e inundaciones que pueden originar la pérdida no sólo de cultivos sino también de vidas.

Las Iluvias del Monzón aportan casi el $80 \%$ de la precipitación anual al territorio de la India. Es por esta razón que cumplen un rol fundamental sobre la agricultura de este país. El retardo del Monzón puede provocar sequías prolongadas.

La actividad agrícola de la India es la principal dentro de las actividades económicas del país, es por ello que realizar un análisis del impacto del monzón sobre ella puede ser importante para poder lograr una correcta planificación de dicha actividad, teniendo en cuenta los efectos negativos que se desencadenan a partir del fenómeno del Monzón sobre la población.

Es por ello, que sería pertinente actuar de manera conjunta con los agricultores que, en definitiva, son los actores más perjudicados por las variaciones climáticas. 


\section{Anexo}

Tabla de datos pluviométricos durante el monzón de verano sobre los años comprendidos entre 1901 y 2013 , para las regiones Norte, Noreste, Centro y Sur de la India.

HOMOGENEOUS REGIONS JUNE-SEPT RAINFALL

\begin{tabular}{|c|c|c|c|c|}
\hline \multicolumn{5}{|c|}{ ACTUAL RAINFALL IN MM } \\
\hline YEAR & NWI & NEI & $\mathrm{Cl}$ & SPIN \\
\hline 1901 & 542.7 & 1156.3 & 863.3 & 622.9 \\
\hline 1902 & 555.1 & 1317.2 & 850 & 686.7 \\
\hline 1903 & 680.9 & 1160.6 & 987.1 & 859.9 \\
\hline 1904 & 611.7 & 1243 & 806.3 & 603.5 \\
\hline 1905 & 449.6 & 1383.1 & 783.4 & 560.6 \\
\hline 1906 & 732.7 & 1253.7 & 1009.9 & 726.5 \\
\hline 1907 & 450.7 & 1263.9 & 906 & 736.8 \\
\hline 1908 & 787.3 & 1113.8 & 1071 & 767.7 \\
\hline 1909 & 831.8 & 1381.8 & 946 & 729 \\
\hline 1910 & 725.2 & 1365.3 & 1054.3 & 773.4 \\
\hline 1911 & 480.2 & 1387.7 & 809 & 588.9 \\
\hline 1912 & 615 & 1135.2 & 950.4 & 737.1 \\
\hline 1913 & 468.8 & 1385 & 938.8 & 592.5 \\
\hline 1914 & 765.6 & 1196.4 & 1119.9 & 836.1 \\
\hline 1915 & 550 & 1263.1 & 844.8 & 755.9 \\
\hline 1916 & 834.2 & 1473.1 & 1077.5 & 822.6 \\
\hline 1917 & 1024 & 1433.6 & 1174.7 & 808.3 \\
\hline 1918 & 345.4 & 1679.6 & 679.9 & 434 \\
\hline 1919 & 691.3 & 1412.5 & 1119.7 & 692.1 \\
\hline 1920 & 504.8 & 1383.8 & 760.1 & 625.3 \\
\hline 1921 & 675.8 & 1539.1 & 999.8 & 742.3 \\
\hline 1922 & 770.7 & 1600.9 & 954.6 & 611.3 \\
\hline 1923 & 640.8 & 1334.5 & 934.3 & 769.8 \\
\hline 1924 & 749.5 & 1505.5 & 883 & 865.2 \\
\hline 1925 & 684.6 & 1324.7 & 939.8 & 670.9 \\
\hline 1926 & 733.1 & 1416.4 & 1115.3 & 694.3 \\
\hline 1927 & 656 & 1440.8 & 1030.7 & 738.8 \\
\hline 1928 & 469.1 & 1464.1 & 962.4 & 650.4 \\
\hline 1929 & 575.4 & 1394.8 & 930.3 & 667.7 \\
\hline 1930 & 612.6 & 1383.5 & 969.3 & 592.8 \\
\hline 1931 & 656.3 & 1396.4 & 1038.4 & 778 \\
\hline 1932 & 594.1 & 1345.4 & 998.2 & 677.6 \\
\hline 1933 & 770.6 & 1448.4 & 1202.7 & 854.1 \\
\hline 1934 & 680.8 & 1413.6 & 1156.7 & 651.3 \\
\hline
\end{tabular}


Análisis del impacto del fenómeno del Monzón en la producción agrícola de la India

\begin{tabular}{|c|c|c|c|c|}
\hline 1935 & 581.6 & 1502.8 & 988 & 693.9 \\
\hline 1936 & 774.9 & 1506.5 & 1025.9 & 718.7 \\
\hline 1937 & 586.4 & 1338.1 & 1096.6 & 637.2 \\
\hline 1938 & 613.7 & 1533.8 & 1054.3 & 839.6 \\
\hline 1939 & 518.4 & 1461.1 & 939.3 & 627.6 \\
\hline 1940 & 564.9 & 1270.3 & 1060.8 & 742.1 \\
\hline 1941 & 506.6 & 1458.6 & 806.4 & 652.5 \\
\hline 1942 & 826.3 & 1469.1 & 1165.8 & 718.9 \\
\hline 1943 & 712.9 & 1403.4 & 1050 & 687.1 \\
\hline 1944 & 678.5 & 1345.5 & 1155 & 648.3 \\
\hline 1945 & 690.2 & 1297.1 & 1116 & 677.7 \\
\hline 1946 & 651.2 & 1389 & 1143.4 & 784.9 \\
\hline 1947 & 663.6 & 1405.7 & 1052.2 & 851 \\
\hline 1948 & 732.8 & 1474 & 962.4 & 736.4 \\
\hline 1949 & 593.5 & 1479.6 & 999.8 & 806.6 \\
\hline 1950 & 785.4 & 1361.2 & 955.5 & 769.5 \\
\hline 1951 & 433.2 & 1204.5 & 798.3 & 699.4 \\
\hline 1952 & 642.5 & 1328.1 & 917.4 & 528.4 \\
\hline 1953 & 777.8 & 1483.7 & 1008.9 & 829.8 \\
\hline 1954 & 610.9 & 1335.6 & 1082.3 & 788.7 \\
\hline 1955 & 736.3 & 1317.7 & 1135.4 & 755.5 \\
\hline 1956 & 734.6 & 1419 & 1141.9 & 811.2 \\
\hline 1957 & 753.7 & 1250.7 & 866.4 & 732 \\
\hline 1958 & 749.9 & 1286.1 & 1120.3 & 813.7 \\
\hline 1959 & 653.9 & 1338.3 & 1207.2 & 951.7 \\
\hline 1960 & 631.9 & 1452.4 & 965.4 & 732 \\
\hline 1961 & 804.2 & 1229.9 & 1294.1 & 915.7 \\
\hline 1962 & 650.2 & 1255.6 & 881.9 & 784.4 \\
\hline 1963 & 624.6 & 1328.5 & 1002.3 & 689.4 \\
\hline 1964 & 685.3 & 1490.2 & 1095 & 843.7 \\
\hline 1965 & 411.5 & 1327.8 & 764.6 & 668.6 \\
\hline 1966 & 587.2 & 1168 & 786.9 & 677.3 \\
\hline 1967 & 669.8 & 1172.8 & 1026.1 & 707.1 \\
\hline 1968 & 508.3 & 1300.8 & 860.4 & 674.9 \\
\hline 1969 & 555.2 & 1462.9 & 1022.8 & 660.4 \\
\hline 1970 & 669.3 & 1474.8 & 1176 & 773.6 \\
\hline 1971 & 690.4 & 1405.9 & 998.4 & 638.8 \\
\hline 1972 & 412.8 & 1106.3 & 754.9 & 573.3 \\
\hline 1973 & 663.7 & 1380.1 & 1084.8 & 686.2 \\
\hline 1974 & 478.9 & 1499.6 & 703.2 & 721.4 \\
\hline
\end{tabular}




\begin{tabular}{|c|c|c|c|c|}
\hline 1975 & 855.5 & 1142.4 & 1095.3 & 922.2 \\
\hline 1976 & 714.8 & 1271.9 & 997.7 & 667.6 \\
\hline 1977 & 686.7 & 1387.4 & 981.8 & 655.7 \\
\hline 1978 & 762.1 & 1371.2 & 970.7 & 860 \\
\hline 1979 & 356.2 & 1216.7 & 783.9 & 637.1 \\
\hline 1980 & 698.4 & 1277.5 & 1024.5 & 669.7 \\
\hline 1981 & 577 & 1299.5 & 949.9 & 789.5 \\
\hline 1982 & 500.9 & 1144.3 & 847.3 & 596.2 \\
\hline 1983 & 689.1 & 1279 & 1124.5 & 910.4 \\
\hline 1984 & 609.5 & 1526.3 & 855.6 & 562.5 \\
\hline 1985 & 618.7 & 1347.1 & 867.2 & 562.8 \\
\hline 1986 & 544.2 & 1196.5 & 822.4 & 594.3 \\
\hline 1987 & 371.4 & 1684.2 & 683.6 & 541.9 \\
\hline 1988 & 808.1 & 1551.3 & 1065.6 & 901.8 \\
\hline 1989 & 592.6 & 1466 & 906 & 832.7 \\
\hline 1990 & 684.3 & 1416.4 & 1112.1 & 717.4 \\
\hline 1991 & 450.4 & 1299.8 & 916.5 & 820 \\
\hline 1992 & 623.8 & 1054 & 955.4 & 863.8 \\
\hline 1993 & 559.3 & 1474.5 & 1069.8 & 652.4 \\
\hline 1994 & 729.9 & 1131.3 & 1349.4 & 705.2 \\
\hline 1995 & 696.4 & 1471 & 867.6 & 722.2 \\
\hline 1996 & 749.1 & 1291.1 & 933.9 & 875.5 \\
\hline 1997 & 660 & 1473.2 & 994.1 & 728.7 \\
\hline 1998 & 643.1 & 1468.4 & 937.2 & 863.4 \\
\hline 1999 & 559.8 & 1508.5 & 944.3 & 628.8 \\
\hline 2000 & 580.3 & 1386.3 & 790.3 & 801.3 \\
\hline 2001 & 561.6 & 1210.2 & 955.1 & 659.4 \\
\hline 2002 & 434.7 & 1353.9 & 829.2 & 506.7 \\
\hline 2003 & 663.7 & 1300.4 & 1096.6 & 648 \\
\hline 2004 & 481.4 & 1211.6 & 889.1 & 617.4 \\
\hline 2005 & 549.3 & 1043.7 & 1097.6 & 807 \\
\hline 2006 & 574.9 & 1160.7 & 1157.3 & 684.5 \\
\hline 2007 & 521.9 & 1502 & 1075.8 & 902.1 \\
\hline 2008 & 641.2 & 1326.9 & 958.7 & 692.5 \\
\hline 2009 & 394.6 & 1098.1 & 795.5 & 682.3 \\
\hline 2010 & 686.8 & 1172.7 & 1028.8 & 848.1 \\
\hline 2011 & 655 & 1255 & 1068.1 & 713.2 \\
\hline 2012 & 567.7 & 1305.5 & 934 & 644 \\
\hline 2013 & 672 & 1045.5 & 1193.8 & 825.7 \\
\hline
\end{tabular}

Fuente: Indian Meteorological Department (2015) 


\section{Referencias Bibliográficas}

ARCGIS. Recuperado de: https://www.arcgis.com

BHALLA, G.S. (1985). Desarrollo agrícola de la India desde su independencia. Estudios de Asia y Africa. (vol. 20) 4,603614. Recuperado de:

http://codex.colmex.mx:8991/exlibris/aleph/a18 1/apache media/NRDR7GEPAGPUFXHG532C4X4MKJ1JAV.pdf BARRY, R. G. y CHORLEY, R. J. (1999). Tiempo y clima en los trópicos. En Atmósfera, tiempo y clima. Barcelona: Omega. pp. 253-309.CAMACHO RUIZ, J.L. (2008). El fenómeno del Niño y la variabilidad climática en América del Sur. AME Boletín. 19, 36-39. Recuperado de: http://pkp.ame-web.org/index.php/TyC/article/viewFile/400/396 CENTRO DE DERECHOS HUMANOS Y JUSTICIA SOCIAL. (11/05/2011). Cada 30 Minutos: Granjero Los suicidios y la crisis agraria en la India. [Mensaje de Blog]. Recuperado de: https://foodfreedom.wordpress.com/2011/05/12/every-30minutes-farmer-suicides-and-the-agrarian-crisis-in-india/\#more-8104

DUPUIS, J. (1975). El espacio indio. En Asia meridional (tercera parte. cap. 1). Barcelona: Ariel. pp. 169-246.

FÜHRER, M. (14/05/2013). Los peligros de la "Revolución Verde" en India. [Mensaje de Blog]. Recuperado de: http://www.ecoagricultor.com/revolucion-verde-india-pesticidas-salud/

GÓMEZ, T. y ROMANILLOS, P. (2012). Los elementos del clima. En El cambio climático. Pasado, presente y futuro de un mundo nuevo (cap. 3). Barcelona: Océano. pp. 72-160.

GOUROU, P. (1976). Extensión y diversidad del espacio indostánico. En Asia. (quinta parte. Cap. 23). Barcelona: Labor. pp.337-390.

HOYOS ORTIZ, C.D. (2003). Diagnóstico, Modelación y Predicción del Monzón Sur-Asiático y su Variabilidad Intraestacional (Tesis de maestría). Universidad Nacional de Colombia. Medellín. Recuperado de: http://www.bdigital.unal.edu.co/12133/

INDIAN METEOROLOGICAL DEPARTMENT. Recuperado de:

http://www.imd.gov.in/section/nhac/dynamic/Monsoon frame.htm

LA INDIA: La soberanía alimentaria en en el Estado de Tamil Nadu. Recuperado de:

http://www.siemenpuu.org/es/programme/tamil-nadu

ORDÓÑEZ PÉREZ, P. (2013). Principales fuentes de humedad para la India y desarrollo de fenómenos de precipitación a escala sinóptica (Tesis de doctorado).Universidad Pablo de Olavide. Sevilla. Recuperado de:

file://C:/Users/Usuario/Downloads/paulina ordonez tesis\%20(1).pdf

ORGANIZACIÓN PANAMERICANA DE LA SALUD (2000). Fenómeno El Niño, 1997-1998. SerieCrónicas de Desastres. 8, 1-288. Recuperado de: www.paho.org/disasters/index.php?gid=1823\&option=com. 
SÁNCHEZ, L. (2001-2002). Cambio climático global. El clima del futuro.. Reflexiones Geográficas.10, 101-123.

VALIENTE, Ó. M.(1996-1997). La cara oculta de El Niño: Anomalías climáticas y ecológicas en el PacíficoOccidental., Revista de Geografía. (vols. 30-31), 97-110. Recuperado de:

www.raco.cat/index.php/RevistaGeografia/article/download/.../56903

---.(1999). Evolución en el estudio del fenómeno ENSO (El Niño- Oscilación del Sur): de anomalía local a la predicción de variaciones climáticas globales., Investigaciones geográficas. 21, 5-20. Recuperado de : file:///C:/Users/Usuario/Downloads/DialnetEvolucionEnEIEstudioDelFenomenoENSOEINinooscilacio-111693.pdf STRAHLER, A. y STRAHLER, A. (1997). Masas de aire y borrascas. En Geografía Física. Barcelona: Omega. pp. 128-146. 